Tạp chí Khoa học và Công nghệ Biển T12 (2012). Số 4. Tr 1 - 14

\title{
NGHIÊN CÚU CHẾ ĐỘ DÒNG CHẢY TẠI VỊNH PHAN THIẾT BẰNG MÔ HİNH BA CHIỀU PHI TUYẾN VỚI PHƯंƠNG PHÁP PHẦN TỬ HŨ̉U HẠN
}

\author{
BÙI HỒNG LONG, TRẦN VĂN CHUNG
}

Viện Hải dương học Nha Trang

Tóm tắt: Kết quả nghiên cứu các dạng dòng chảy khác nhau (dòng dux, dòng triều, dòng chảy do gió và dòng tổng hợp) bằng mô hình ba chiều phi tuyến với phuơng pháp phần tử hưu hạn cho vùng biển Phan Thiết đã cho thấy khả năng ứng dụng của phuơng pháp này vào mô hình hóa chế độ dòng chảy tại các vùng nghiên cứu có địa hình phức tạp, biên mở rộng.

Để đánh giá khả năng ứng dụng và kiểm chưng mô hình, chúng tôi đã tính riêng dòng chảy do ảnh huơơng của triều dưới tác động của 5 sóng triều chính $M_{2}, S_{2}, K_{1}, O_{1}, N_{2}$. Các hằng số điều hòa của các sóng triều này sẽ được so sánh với số liệu phân tích tù trạm đo mưc nước Phan Thiết. Theo kết quả so sánh thì sai số tuyệt đối: về biên độ cao nhất là $3,1 \mathrm{~cm}$ (đối với sóng $M_{2}$ ), thấp nhất $0 \mathrm{~cm}$ (chính xác đối với $S_{2}$ ); về pha cao nhất 13,90 (đối với sóng $K_{1}$ ) và thấp nhất $3,8^{0}$ (đối với sóng $S_{2}$ ).

Về dòng chảy tầng mặt, trong trương gió mùa Đông Bắc, tốc độ dòng ngang do gió lớn nhất là $85,3 \mathrm{~cm} / \mathrm{s}$, huớng $185,7^{0}$ xung quanh vị trí có tọa độ $\left(108,0554^{\circ} \mathrm{E} ; 10,7014^{\circ} \mathrm{N}\right)$; nhung với dòng chảy tổng hợp là $60,6 \mathrm{~cm} / \mathrm{s}$, huớng $209,6^{\circ}$; tại vị trí $\left(108,0820^{\circ} \mathrm{E}\right.$; $\left.10,7015^{\circ} \mathrm{N}\right)$. Trong khi đó với truờng gió mùa Tây Nam, tốc độ dòng ngang đạt cực đạt $53 \mathrm{~cm} / \mathrm{s}$, hướng $10,3^{\circ}$; tại vị trí $\left(108,1086^{\circ} \mathrm{E} ; 10,7015^{\circ} \mathrm{N}\right)$. Theo tính toán phân bồ dòng chảy tổng hợp tốc độ dòng ngang đạt cưc là $36,6 \mathrm{~cm} / \mathrm{s}$, huớng $23,8^{0}$; tại vị trí $\left(108,0355^{\circ} \mathrm{E} ; 10,7054^{\circ} \mathrm{N}\right)$.

Tù nhũng kết quả nghiên cứu áp dụng mô hình ba chiều phi tuyến với phuơng pháp phần tử hưu hạn vào đánh giá chế độ dòng chảy tại vịnh Phan Thiết, đã gợi mở một khá năng nghiên cúu chi tiết các phân bố dòng chảy tại khu vực với hy vọng có thể tìm ra các vị trí có phân bố dòng chảy tuơng đối đặc biệt nhằm muc đích cung cấp các thông tin tin cậy cho việc hoạch định và phát triền kinh tế - xã hội biển tại địa phuơng một cách hợp lý.

\section{TÀI LIỆU VÀ PHƯƠNG PHÁP}

Dựa vào các thông tin được cập nhật từ các chuyến khảo sát liên quan đến vùng biển Bình Thuận như cấu trúc nhiệt - muối, trường gió, trường dòng chảy và các thông tin từ bản đồ viễn thám, nhằm cung cấp cơ sở dữ liệu đầy đủ cho tiến hành mô hình hóa quá trình thủy động lực học. Cụ thể, các cập nhật chính $[4,5]$ :

Bản đồ độ sâu được cập nhật từ dự án Nước trồi Nam Trung bộ (hợp tác Việt-Đức).

Thông tin số liệu độ sâu và biên địa hình từ trang web:

http://www.ngdc.noaa.gov/mgg/global/global.html

Số liệu gió: được thu thập từ trang web: ftp://ftp.ssmi.com/qscat/bmaps_v03, với phân tích thống kê các trường gió để tìm vận tốc và hướng gió trung bình theo mùa và theo tháng giai đoạn 1996-2008. Việc nội suy số liệu gió theo phân bố lưới (lưới tam giác trong 
phương pháp phần tử hữu hạn) được thực hiện bằng phần mềm MatlabR2009a.

Số liệu nhiệt - muối: Chúng tôi đã tập trung phân tích dữ liệu tại các nguồn số liệu sau:

Nguồn cơ sở dữ liệu của Viện Hải dương học Nha Trang (VODC)

Từ PHC 3.0 thu thập từ trang web:

http://psc.apl.washington.edu/POLES/PHC2/Climatology.html

http://pacificinfo.ru/ (cơ sở dữ liệu của Nga)

Nguồn số liệu từ các chuyến khảo sát hợp tác Việt-Đức (2003-2006).

Các lớp số liệu nhiệt - muối được sử dụng trong mô hình các nguồn cơ sở dữ liệu nêu trên được phân theo các tầng sau: tầng mặt, $10 \mathrm{~m}, 20 \mathrm{~m}, 30 \mathrm{~m}$.

Sử dụng số liệu gió tại khu vực Phú Quý - Bình Thuận $\left(104^{\circ} 28^{\prime} \mathrm{E} ; 19^{\circ} 16^{\prime} \mathrm{N}\right)$

Để đánh giá tính chất địa phương của các trường gió, chúng tôi sử dụng số liệu gió tại trạm khí tượng Phú Quý (1987-2007), tần suất đo số liệu là 6 tiếng một lần: 1, 7, 13, 19 giờ (hình 1).

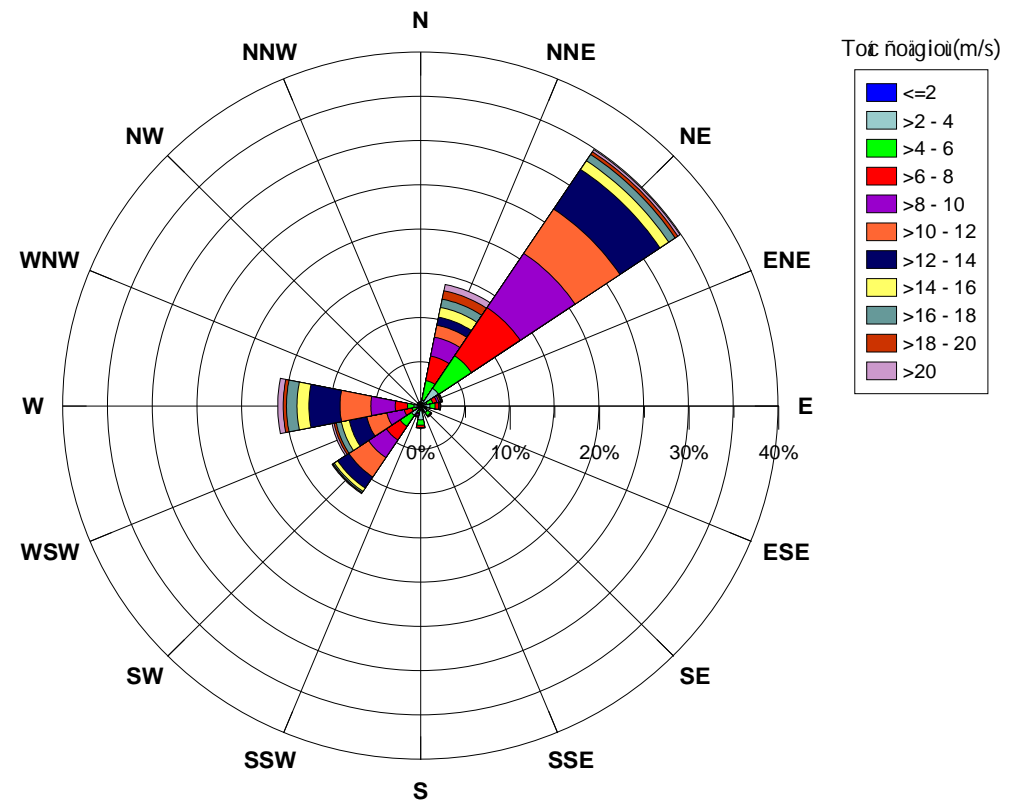

Hình 1. Hoa gió tại khu vực Phú Quý - Bình Thuận

Từ kết quả phân tích hoa gió tại Phú Quý, chúng tôi có những nhận xét sau:

Với mùa gió Đông Bắc (NE), tại địa phương có hai hướng gió chính là hướng Đông Bắc chiếm tần suất $34,83 \%$, đây là hướng xuất hiện nhiều nhất khu vực nghiên cứu, thời gian hướng Đông Bắc $(\mathrm{NE})$ kéo dài có thể đạt 137 ngày và hướng thứ hai là Bắc Đông Bắc $(\mathrm{NNE})$ với tần suất xuất hiện khoảng $14 \%$ và thời gian duy trì cực đại là 49 ngày.

Với mùa gió Tây Nam $(\mathrm{SW})$, có 3 hướng gió đại diện. Hướng Tây $(\mathrm{W})$ với tần suất xuất hiện $15,96 \%$, thời gian duy trì cực đại là 23 ngày; hướng Tây Tây Nam (WSW) với tần suất xuất hiện $10,09 \%$, thời gian duy trì dài nhất là 14 ngày; hướng Tây Nam $(\mathrm{SW})$ với tần suất xuất hiện khoảng $11,19 \%$, có thời gian duy trì cao nhất đạt 57 ngày (duy trì lâu nhất trong trường gió mùa Tây Nam). 


\section{CƠ SỞ MÔ HÌNH THỦY ĐộNG LỰC HỌC}

\section{Các phương trình trong mô hình và phương pháp số trị}

Các phương trình thủy động lực học ba chiều (3-D) với các thừa nhận xấp xỉ Boussinesq và áp suất thủy tĩnh. Nhiệt độ và độ mặn tham gia vào quá trình vận chuyển, mật độ nước biển được xác định từ phương trình trạng thái. Sự tiêu tán ở quy mô lưới nhỏ được thể hiện dưới dạng độ nhớt rối (độ khuếch tán).

Các phuoong trình chủ đạo

Có 6 biến chính trong mô hình 3-D, chúng được thể hiện trong các phương trình sau:

Hai thành phần nằm ngang $(x, y)$ của các phuơng trình động lượng:

$$
\frac{d \vec{v}}{d t}+\vec{f} \times \vec{v}=g \nabla_{x y} \zeta-\frac{\partial}{\partial z}\left(N_{m} \frac{\partial \vec{v}}{\partial z}\right)=-\frac{g}{\rho_{0}} \int_{z}^{\zeta} \nabla_{x y} \rho d z+\vec{F}_{m}+\frac{\sigma}{\rho}\left(\vec{v}_{\sigma}-\vec{v}\right)
$$

Phương trình bảo toàn nhiệt và muối:

$$
\begin{aligned}
& \frac{d T}{d t}-\frac{\partial}{\partial z}\left(N_{h} \frac{\partial T}{\partial z}\right)=F_{T}+\frac{\sigma}{\rho}\left(T_{\sigma}-T\right) \\
& \frac{d S}{d t}-\frac{\partial}{\partial z}\left(N_{h} \frac{\partial S}{\partial z}\right)=F_{S}+\frac{\sigma}{\rho}\left(S_{\sigma}-S\right)
\end{aligned}
$$

Các phưong trình đối với động năng dòng rối và độ dài pha trộn:

$$
\begin{aligned}
\frac{d q^{2}}{d t}-\frac{\partial}{\partial z}\left(N_{q} \frac{\partial q^{2}}{\partial z}\right)= & 2\left[N_{m}\left(\left(\frac{\partial u}{\partial z}\right)^{2}+\left(\frac{\partial v}{\partial z}\right)^{2}\right)+\frac{g}{\rho_{0}} N_{h} \frac{\partial \rho}{\partial z}\right]-2\left[\frac{q^{3}}{B_{1} l}\right]+\frac{\sigma}{\rho}\left(q_{\sigma}^{2}-q^{2}\right)(4) \\
\frac{d q^{2} l}{d t}-\frac{\partial}{\partial z}\left(N_{q} \frac{\partial q^{2} l}{\partial z}\right) & =l E_{1}\left[N_{m}\left(\left(\frac{\partial u}{\partial z}\right)^{2}+\left(\frac{\partial v}{\partial z}\right)^{2}\right)+\frac{g}{\rho_{0}} N_{h} \frac{\partial \rho}{\partial z}\right]-l W\left[\frac{q^{3}}{B_{1} l}\right] \\
& +\frac{\sigma}{\rho}\left(q_{\sigma}^{2} l \sigma-q^{2} l\right)
\end{aligned}
$$

Trong đó: $\mathrm{E}_{1}$ và $\mathrm{B}_{1}$ là các hằng số thực nghiệm theo [11],

$$
\mathrm{W} \text { là một hàm sát tường chắn theo [6]. }
$$

\section{Các điều kiện biên}

Chúng tôi giải bài toán này trên cơ sở các điều kiện biên theo phương nằm ngang được bảo toàn. Theo phương thẳng đứng: đối với vận tốc nằm ngang, ứng suất trượt khí quyểnđược xác định tại bề mặt : $\left.\quad N_{m} \frac{\partial \vec{v}}{\partial z}\right|_{z=\zeta}=H \vec{\Psi}=\vec{\tau}_{w}$

Với: $\quad \vec{\tau}_{w}=C_{d s} \rho_{a} \vec{W}|\vec{W}|$

Trong đó: $\vec{W}$ : Véc tơ vận tốc gió $10 \mathrm{~m}$ trên mặt biển.

$$
\rho_{\mathrm{a}} \text { : Mật độ không khí }\left(\rho_{\mathrm{a}}=1,25 \mathrm{~kg} \cdot \mathrm{m}^{-3}\right)
$$


Chúng tôi sử dụng hệ số cản phi tuyến (dựa trên công thức Large và Pond (1981) [9] được hiệu chỉnh đối với tốc độ gió thấp theo Trenberth và cộng sự (1990) [21]):

$$
C_{d s}=\left\{\begin{array}{l}
0,00218 \text { cho }:|\overrightarrow{\mathrm{v}}| \leq 1(\mathrm{~m} / \mathrm{s}) \\
\left(0,62+\frac{1,56}{|\vec{v}|}\right) \times 10^{-3} \text { cho }: 1(\mathrm{~m} / \mathrm{s})<|\overrightarrow{\mathrm{v}}|<3(\mathrm{~m} / \mathrm{s}) \\
0,00114 \text { cho }: 3(\mathrm{~m} / \mathrm{s}) \leq|\overrightarrow{\mathrm{v}}|<10(\mathrm{~m} / \mathrm{s}) \\
(0,49+0,065|\overrightarrow{\mathrm{v}}|) \times 10^{-3} \text { cho }:|\overrightarrow{\mathrm{v}}| \geq 10(\mathrm{~m} / \mathrm{s})
\end{array}\right.
$$

Tại đáy chúng ta sử dụng điều kiện trượt bậc hai thông thường quan hệ ứng suất trượt tới vận tốc đáy $\vec{v}_{b}$ qua hệ số cản ứng suất đáy bậc hai không thứ nguyên, $\mathrm{C}_{\mathrm{d}}$

$$
\left.N_{m} \frac{\partial \vec{v}}{\partial z}\right|_{z=-h}=C_{d}\left|\vec{v}_{b}\right| \vec{v}_{b}=k \vec{v}_{b}
$$

trong đó: $\mathrm{k}$ - hệ số ứng suất đáy tuyến tính.

Đưa vào nhiệt độ không khí được xác định tại bề mặt như "kiểu III" hoặc điều kiện bức xạ với tốc độ làm nóng $\alpha$ và nhiệt độ cân bằng $\mathrm{T}_{0}$ :

$$
\left.N_{h} \frac{\partial T}{\partial z}\right|_{z=\zeta}=-\alpha\left(T-T_{0}\right)
$$

Tại đáy, thông lượng nhiệt được giả sử là không đáng kể

$$
\left.N_{h} \frac{\partial T}{\partial z}\right|_{z=-h}=0
$$

Tương tự, các điều kiện không thông lượng được áp đặt trên $S$ tại bề mặt và đáy. Đối với $\mathrm{q}^{2}$, các điều kiện Dirichlet là đòi hỏi tại đáy:

$$
q^{2}=B_{1}^{2 / 3} u_{*}^{2}
$$

với vận tốc ma sát $u_{*}^{2}=\left|N_{m} \frac{\partial \vec{v}}{\partial z}\right|$ xác định theo các điều kiện biên vận tốc. Phù hợp với điều kiện trượt tại đáy, biên được tính toán $z_{b}=-h$ là vị trí trong phạm vi lớp ứng suất không đổi tại một độ cao $\xi_{b}=1 \mathrm{~m}$ trên nền đáy thực tế. Độ dài pha trộn được tính toán tại đáy được đặt cho phù hợp với quy luật của tường chắn:

$$
I=\kappa \xi_{b}
$$

trong đó $\kappa=0,4$ là hằng số Von Karman. Tại bề mặt tự do, các điều kiện không thông lượng (Neuman) được ứng dụng tới cả hai $\mathrm{q}^{2}$ và $\mathrm{q}^{2} l$. Hàm giả định sát tường chắn $\mathrm{W}$ được lấy từ [6] và được kết hợp chặt chẽ tính bất đối xứng giữa bề mặt và đáy "các tường chắn": $\quad W=1+E_{2}\left[\frac{1}{\kappa\left(z-z_{b}+\xi_{b}\right)}\right]^{2}+E_{3}\left[\frac{1}{\kappa\left(\zeta-z+\xi_{s}\right)}\right]^{2}$ với $\frac{l}{\kappa d}$ cách tiếp cận đồng nhất ở khoảng cách $\mathrm{d}$ thì một trong hai tường chắn được loại 
bỏ. Cả hai $\xi_{b}$ và $\xi_{s}$ được đặt bằng 1 . Sau cùng, điều kiện động học được áp đặt trên vận tốc thẳng đứng $\mathrm{w}$ tại bề mặt:

$$
w=\frac{\partial \zeta}{\partial t}+\vec{v} \cdot \nabla_{x y} \zeta-(P-E)
$$

và tại đáy:

$$
w=-\vec{V} \cdot \nabla_{x y} h
$$

Cả hai được áp đặt như các điều kiện Dirichlet trên đạo hàm - $\mathrm{z}$ theo [10].

Các ký hiệu sủ dụng trong các phuoong trình trên:

$\vec{v}(x, y, z, t)$ - vận tốc dòng, với các thành phần trong tọa độ Đề-các $(\mathrm{u}, \mathrm{v}, \mathrm{w}) ; \overline{\vec{v}}(x, y, t)$ trung bình thẳng đứng của $\vec{v} ; \zeta(x, y, t)$ - độ cao bề mặt tự do; $h(x, y)$ - độ sâu biển (chính xác hơn, độ sâu của vị trí theo lớp ứng suất đáy không đổi mà tại điều kiện biên được ứng dụng, điển hình khoảng $1 \mathrm{~m}$ trên nền đáy); $\mathrm{H}(\mathrm{x}, \mathrm{y}, \mathrm{t})$ - tổng độ sâu, $\mathrm{H}=\mathrm{h}+\zeta ; \rho(x, y, z, t)$ mật độ nước biển, $\rho_{0}$ là giá trị trung bình; $\mathrm{T}(\mathrm{x}, \mathrm{y}, \mathrm{z}, \mathrm{t})$ - nhiệt độ nước biển; $\mathrm{S}(\mathrm{x}, \mathrm{y}, \mathrm{z}, \mathrm{t})$ - độ mặn nước biển; $\mathrm{q}^{2}(\mathrm{x}, \mathrm{y}, \mathrm{z}, \mathrm{t}) / 2$ - động năng dòng rối; $l(\mathrm{x}, \mathrm{y}, \mathrm{z}, \mathrm{t})$ - độ dài pha trộn dòng rối; $N_{m}(x, y, z, t)$ - độ nhớt rối thẳng đứng; $N_{h}(x, y, z, t)$ - độ khuếch tán rối thẳng đứng đối với nhiệt độ và độ mặn; $N_{q}(x, y, z, t)$ - độ khuếch tán rối thẳng đứng đối với $\mathrm{q}^{2}$ và $\mathrm{q}^{2} 1$; $\vec{F}_{m}, F_{T}, F_{S}$ - là các trao đổi nằm ngang không bình lưu của động năng, nhiệt độ và độ mặn; $\mathrm{g}$ - gia tốc trọng trường; $\vec{f}$ là véc tơ Coriolis, có hướng theo phương thẳng đứng với độ lớn $\mathrm{f} ; \nabla$ - toán tử gradient, $\nabla_{x y}$ là phần nằm ngang của nó, $\frac{d}{d t}$ - đạo hàm toàn phần theo thời gian, cho chuyển động ba chiều của chất lỏng, $\frac{d}{d t}=\frac{\partial}{\partial t}+\vec{v} \cdot \nabla ;(\mathrm{x}, \mathrm{y})$ - các tọa độ Đềcác nằm ngang, chiều $\mathrm{x}$ dương về phía Đông, chiều $\mathrm{y}$ dương về phía Bắc; $\mathrm{z}$ - tọa độ theo phương thẳng đứng, có chiều dương hướng lên; $-h \leq z \leq \zeta ; \mathrm{t}$ - thời gian; $\vec{\tau}_{w}(x, y, t)$ - ứng suất gió tại bề mặt tự do; $\vec{v}_{b}(x, y, z, t)$ - vận tốc dòng chảy nằm ngang tại đáy của cột nước; $\mathrm{C}_{\mathrm{d}}$ - hệ số cản đáy.

Ký hiệu nguồn:

$\sigma(x, y, z, t)$ - nguồn khối lượng phân bố (khối lượng / thời gian / thể tích đơn vị); $\sigma / \rho^{-}$ nguồn theo thể tích (thể tích/thời gian/thể tích đơn vị); $\vec{v}_{\sigma}, T_{\sigma}, S_{\sigma}, q_{\sigma}^{2}, q^{2} l_{\sigma}$ - là tính chất của nguồn lưu chất; $\mathrm{P}$ là lượng mưa tại bề mặt tự do: thể tích/ thời gian/ diện tích đơn vị; $\mathrm{E}$ là lượng bay hơi tại bề mặt tự do: thể tích/thời gian/diện tích đơn vị.

\section{Phương pháp lọc tính dòng dư do triều}

Trong thủy động lực học ven biển, dòng dư thường được xác định là dòng chảy trung bình thu được từ trung bình trong một khoảng thời gian lớn hơn nhiều so với chu kỳ của các thành phần sóng nhật triều và bán nhật triều chính [17]. Các thông tin về hướng và giá trị của dòng dư được quan tâm trong các nghiên cứu về thủy sản, chất lượng nước, vận 
chuyển trầm tích và sinh thái biển. Đây là lý do chính vì sao dòng dư đã luôn được coi là đối tượng quan tâm lớn với nghiên cứu thực nghiệm $[18,22]$ và nghiên cứu số trị [15]. Hiểu biết về dòng dư cũng rất quan trọng cho việc ước tính phân kỳ của thông lượng triều và tiêu tán năng lượng do triều.

Các biến phụ thuộc (mực nước biển và vận tốc dòng) được khai triển dưới dạng một nửa trung bình cộng của các thành phần tuần hoàn của các tần số được biết từ lý thuyết thủy triều [23]:

$$
\begin{aligned}
& \zeta_{z}(x, y, t)=\zeta_{0_{z}}(x, y)+\frac{1}{2} \sum_{n=-N}^{N} \zeta_{n_{z}}(x, y) \exp \left(i \omega_{n} t\right) \\
& u_{z}(x, y, t)=u_{0_{z}}(x, y)+\frac{1}{2} \sum_{n=-N}^{N} u_{n_{z}}(x, y) \exp \left(i \omega_{n} t\right) \\
& v_{z}(x, y, t)=v_{0_{z}}(x, y)+\frac{1}{2} \sum_{n=-N}^{N} v_{n_{z}}(x, y) \exp \left(i \omega_{n} t\right)
\end{aligned}
$$

Trong đó: $\zeta_{\mathrm{z}}$ là dao động mực nước tại các lớp độ sâu, $\left(\mathrm{u}_{\mathrm{z}}, \mathrm{v}_{\mathrm{z}}\right)$ là vận tốc dòng trung bình tại các lớp độ sâu, $\omega_{\mathrm{n}}$ là tần số góc, $\mathrm{t}$ là thời gian, $\mathrm{n}$ là chỉ số cho các thành phần triều, chỉ số dưới “" 0 “ quy định thành phần dư, và các biên độ và tần số điều hòa thỏa mãn:

$$
\omega_{-n}=-\omega_{n} ; \zeta_{-n_{z}}=\zeta{ }_{n_{z}} ; u_{-n_{z}}=u_{n_{z}}^{*} ; v_{-n_{z}}=v_{n_{z}}^{*}
$$

Với “*” biểu thị một liên hợp phức

\section{KẾT QUẢ NGHIÊN CÚ̉U}

\section{Khu vực nghiên cứu}

Để nghiên cứu chi tiết chế độ dòng chảy cho vịnh Phan Thiết, chúng tôi đã thiết lập mạng lưới tính với kinh độ từ $107,997^{\circ} \mathrm{E}$ đến $108,346^{\circ} \mathrm{E}$, vĩ độ từ $10,698^{\circ} \mathrm{N}$ đến $10,963^{\circ} \mathrm{N}$ (hình 2). Mạng lưới tam giác được thiết lập với góc cực tiểu là $30^{\circ}$, diện tích cực đại $514.776 \mathrm{~m}^{2}$, trung bình $69.496 \mathrm{~m}^{2}$, cực tiểu $18.450 \mathrm{~m}^{2}$. Trong đó, diện tích mặt thoáng cho tính toán là $833,8 \mathrm{~km}^{2}$, tương ứng với 6.128 điểm nút nằm ngang và 11.998 lưới tam giác (hình 3). Bài toán được giải theo 15 tầng độ sâu: tầng mặt, $1 \mathrm{~m}, 2 \mathrm{~m}, 3 \mathrm{~m}, 4 \mathrm{~m}, 5 \mathrm{~m}, 6 \mathrm{~m}, 7 \mathrm{~m}$, $8 \mathrm{~m}, 9 \mathrm{~m}, 10 \mathrm{~m}, 15 \mathrm{~m}, 20 \mathrm{~m}, 25 \mathrm{~m}, 30 \mathrm{~m}$. Tầng độ sâu được chúng tôi chọn thể hiện trong báo cáo này các tầng như sau: tầng mặt, tầng $5 \mathrm{~m}$, tầng $10 \mathrm{~m}$.

\section{Kết quả tính cấu trúc dòng triều dư (residual tidal current)}

Xét ảnh hưởng của triều (các ảnh hưởng của yếu tố phi tuyến) lên chế độ dòng chảy của vùng biển Phan Thiết được tính cho 5 sóng triều chính là $\mathrm{M}_{2}, \mathrm{~S}_{2}, \mathrm{~N}_{2}, \mathrm{~K}_{1}, \mathrm{O}_{1}$.

Khi phân tích các ảnh hưởng của triều dư do tác động phi tuyến của các sóng triều riêng rẽ thường không lớn dưới $5 \mathrm{~cm} / \mathrm{s}$ [5]. Tuy nhiên ảnh hưởng dòng dư do tác động tổng hợp của các sóng này lên khu vực nghiên cứu có thể đạt $18,5 \mathrm{~m} / \mathrm{s}$, hướng $182,1^{0}$ tại vị trí $\left(108,3091^{0} \mathrm{E} ; 10,91071^{0} \mathrm{~N}\right), \mathrm{H}=15,5 \mathrm{~m}$ với tốc độ dòng từ Đông sang Tây là khá nhỏ, chỉ đạt $0,7 \mathrm{~cm} / \mathrm{s}$, tốc độ dòng từ Bắc tới $\mathrm{Nam}$ đạt $18,5 \mathrm{~cm} / \mathrm{s}$. Theo kết quả tính, dòng chảy từ Tây sang Đông mạnh nhất là $6,3 \mathrm{~cm} / \mathrm{s}$, tại vị trí $\left(108,0344^{0} \mathrm{E} ; 10,7359^{\circ} \mathrm{N}\right)$; trong khi tốc độ dòng từ Đông sang Tây đạt cực đại là $9,1 \mathrm{~cm} / \mathrm{s}$ vị trí có vận tốc dòng dư đạt $9,2 \mathrm{~cm} / \mathrm{s}$, 
hướng $275,9^{0}\left(108,2814^{0} \mathrm{E} ; 10,8863^{0} \mathrm{~N}\right), \mathrm{H}=16,3 \mathrm{~m}$. Tốc độ dòng từ Nam tới Bắc đạt cực đại là $7,9 \mathrm{~cm} / \mathrm{s}$ tại vị trí mà dòng dư đạt $8,6 \mathrm{~cm} / \mathrm{s}$, hướng $336,4^{0}\left(108,0341^{0} \mathrm{E} ; 10,7100^{\circ} \mathrm{N}\right.$, $\mathrm{H}=15,1 \mathrm{~m})$ và tốc độ dòng cực đại từ Bắc tới $\mathrm{Nam}$ đạt $18,5 \mathrm{~cm} / \mathrm{s}$ tại vị trí có vận tốc dòng dư đạt $18,5 \mathrm{~cm} / \mathrm{s}$, hướng $182,1^{0}\left(108,3091^{0} \mathrm{E} ; 10,91071^{0} \mathrm{~N}\right), \mathrm{H}=15,5 \mathrm{~m}$ (hình 3).

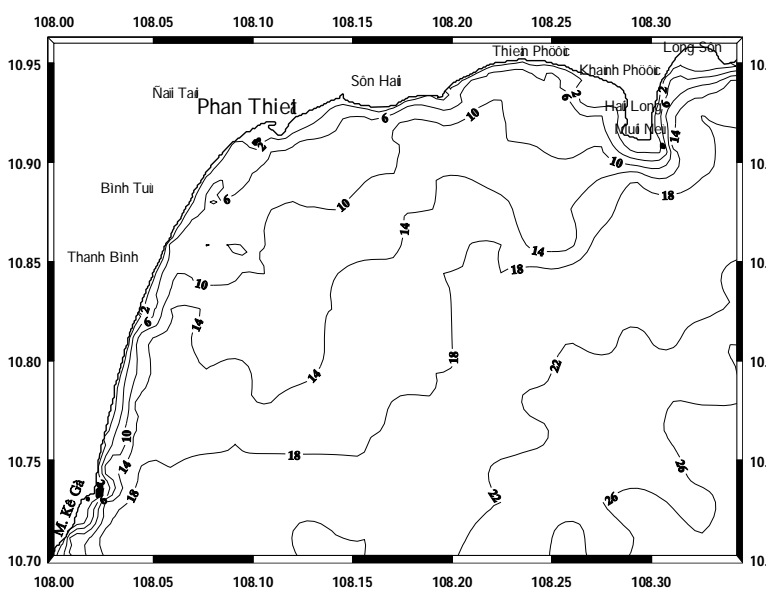

Hình 2. Sơ đồ độ sâu ( $\mathrm{m}$ tính theo mức triều trung bình) vùng nghiên cứu

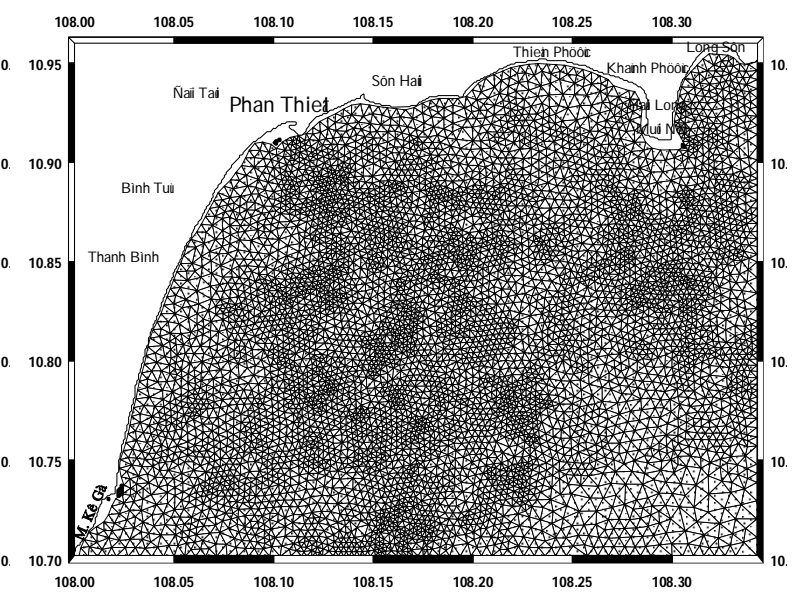

Hình 3. Sơ đồ mạng lưới tam giác dùng để tính toán dòng chảy cho vùng biển Phan Thiết

Trong kết quả nghiên cứu trình bày trong hình 2 , hình 3 , chúng tôi nhận thấy có xuất hiện hai xoáy nghịch (cùng chiều kim đồng hồ) tại vị trí $\left(108,0565^{\circ} \mathrm{E}, 10,7214^{0} \mathrm{~N}\right)$ gần mũi Kề Gà và một xoáy nhỏ hơn tại vị trí $\left(108,2806^{\circ} \mathrm{E}, 10,9011^{\circ} \mathrm{N}\right)$ gần Mũi Né. Vấn đề xuất hiện dòng xoáy này có thể do ảnh hưởng tính phi tuyến của sự thay đổi địa hình ven bờ, tuy nhiên vấn đề này cần phải nghiên cứu chi tiết để có câu trả lời hợp lý.

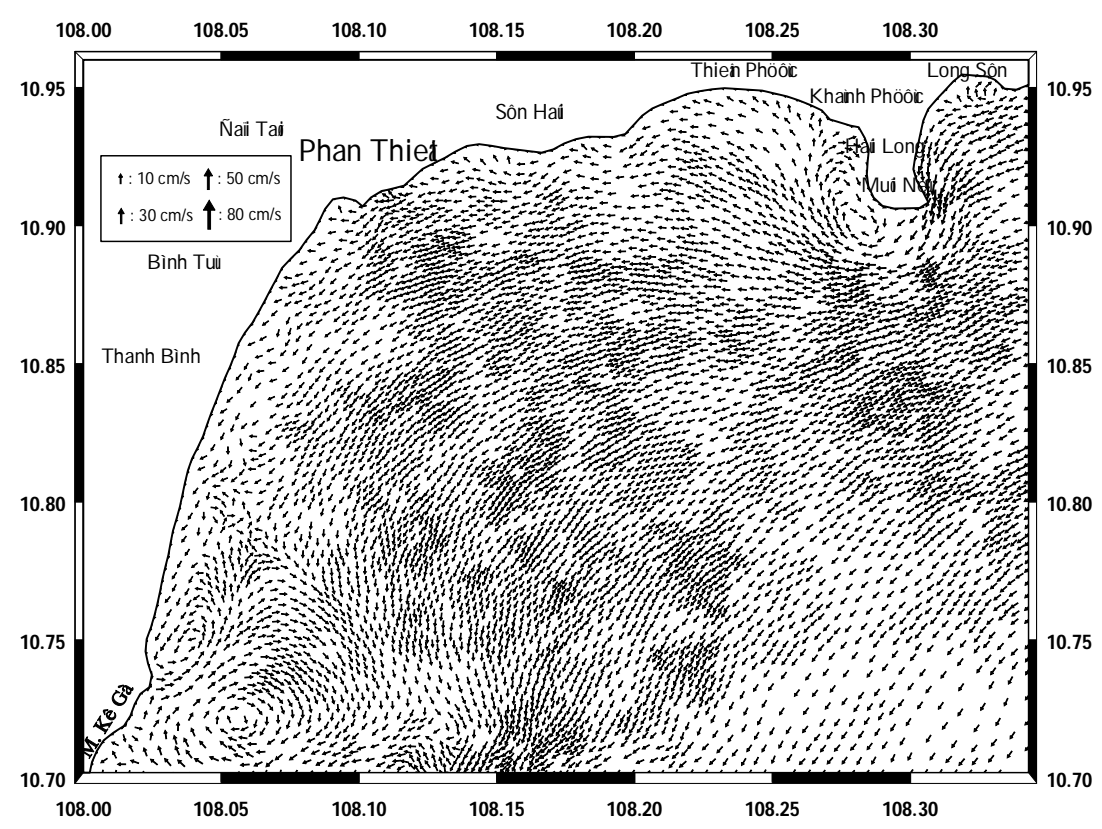

Hình 4. Phân bố triều dư trung bình theo độ sâu do ảnh hưởng của triều tổng hợp 
Bảng 1: So sánh kết quả tính với số liệu đo đạc tại trạm đo thủy triều Phan Thiết

\begin{tabular}{|c|c|c|c|c|c|c|c|c|}
\hline \multirow{2}{*}{$\begin{array}{c}\text { Kinh độ } \\
\left({ }^{0} \mathbf{E}\right)\end{array}$} & \multirow{2}{*}{$\begin{array}{l}\text { Vĩ độ } \\
\left({ }^{0} \mathbf{N}\right)\end{array}$} & \multirow{2}{*}{$\begin{array}{l}\text { Sóng } \\
\text { triều }\end{array}$} & \multicolumn{3}{|c|}{ Biên độ (cm) } & \multicolumn{3}{|c|}{ Pha GMT (độ) } \\
\hline & & & $\begin{array}{c}\text { Thực } \\
\text { đo }\end{array}$ & $\begin{array}{l}\text { Mô } \\
\text { hình }\end{array}$ & $\begin{array}{l}\text { Sai số trị } \\
\text { tuyệt đối }\end{array}$ & $\begin{array}{l}\text { Thực } \\
\text { đo }\end{array}$ & $\begin{array}{l}\text { Mô } \\
\text { hình }\end{array}$ & $\begin{array}{l}\text { Sai số trị } \\
\text { tuyệt đối }\end{array}$ \\
\hline \multirow{5}{*}{107,98} & \multirow{5}{*}{10,70} & $\mathrm{M}_{2}$ & 36,4 & 33,3 & 3,1 & 143,9 & 150,1 & 6,2 \\
\hline & & $\mathrm{S}_{2}$ & 15,8 & 15,8 & 0 & 188,0 & 184,2 & 3,8 \\
\hline & & $\mathrm{K}_{1}$ & 45,3 & 44,9 & 0,4 & 182,3 & 196,2 & 13,9 \\
\hline & & $\mathrm{O}_{1}$ & 37,7 & 35,3 & 2,4 & 146,6 & 155,5 & 8,9 \\
\hline & & $\mathrm{N}_{2}$ & 5,6 & 8,3 & 2,7 & 148,1 & 139,0 & 9,1 \\
\hline
\end{tabular}

\section{Tác động của trường gió mùa lên chế độ dòng chảy tại vịnh Phan Thiết}

\section{a. Đối với trường gió mùa Đông Bắc}

Tại tầng mặt:

Theo tính toán thống kê trung bình, trong toàn bộ vùng nghiên cứu, tốc độ gió cực đại có thể đạt $10,4 \mathrm{~m} / \mathrm{s}$, hướng $228,2^{0}$ (tại vị trí $108,3427^{\circ} \mathrm{E} ; 10,7019^{\circ} \mathrm{N}$ ), trung bình $10,8 \mathrm{~m} / \mathrm{s}$ và cực tiểu $9,1 \mathrm{~m} / \mathrm{s}$, hướng $233,8^{0}$ (tại vị trí $108,0721^{0} \mathrm{E} ; 10,8847^{0} \mathrm{~N}$ ). Theo tính toán phân bố dòng chảy tại tẩng mặt, tốc độ dòng ngang đạt cực đạt $85,3 \mathrm{~cm} / \mathrm{s}$, hướng $185,7^{0}$; tại vị trí $\left(108,0554^{0} \mathrm{E} ; 10,7014^{0} \mathrm{~N}\right)$; độ sâu $2,5 \mathrm{~m}$; độ nhớt rối nằm ngang $\mathrm{A}_{\mathrm{H}}=23,594 \mathrm{~m}^{2} / \mathrm{s}$, nhiệt độ $\mathrm{T}=24,51^{\circ} \mathrm{C}$, độ mặn $\mathrm{S}=33,56 \%$. Trong khi đó, dòng chảy tổng hợp do ảnh hưởng của dòng gió và triều dư có thể đạt: tốc độ dòng ngang đạt cực đạt $60,6 \mathrm{~cm} / \mathrm{s}$, hướng $209,6^{0}$; tại vị trí $\left(108,0820^{\circ} \mathrm{E} ; 10,7015^{\circ} \mathrm{N}\right)$; độ sâu $27,1 \mathrm{~m}$. Rõ ràng, khi phân tích dòng tổng hợp, các ảnh hưởng của tính phi tuyến do tác động của các triều (triều dư) là đáng kể đến sự suy giảm tốc độ và hướng dòng do chỉ chịu tác động của gió mùa thuần túy chi tiết trên hình $5 a, 5 b$.

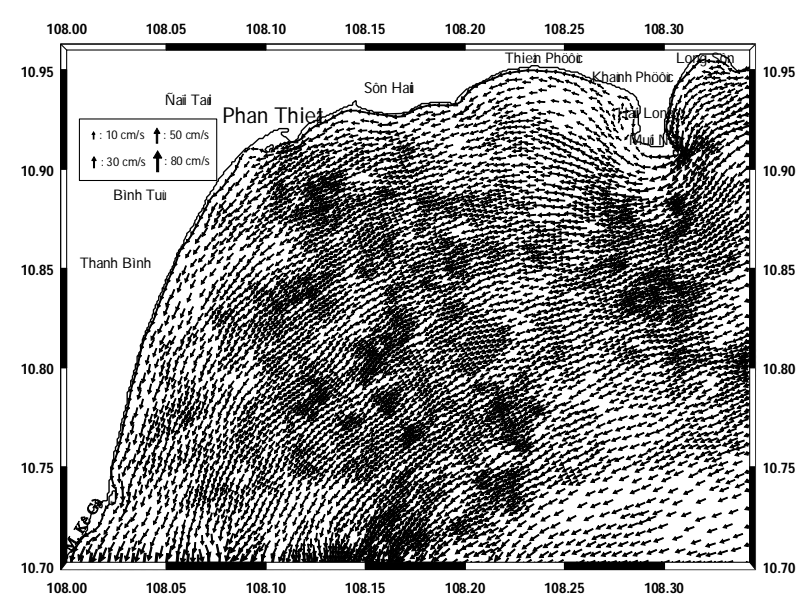

Hình 5a. Phân bố dòng chảy tại tầng mặt vào trường gió mùa Đông Bắc

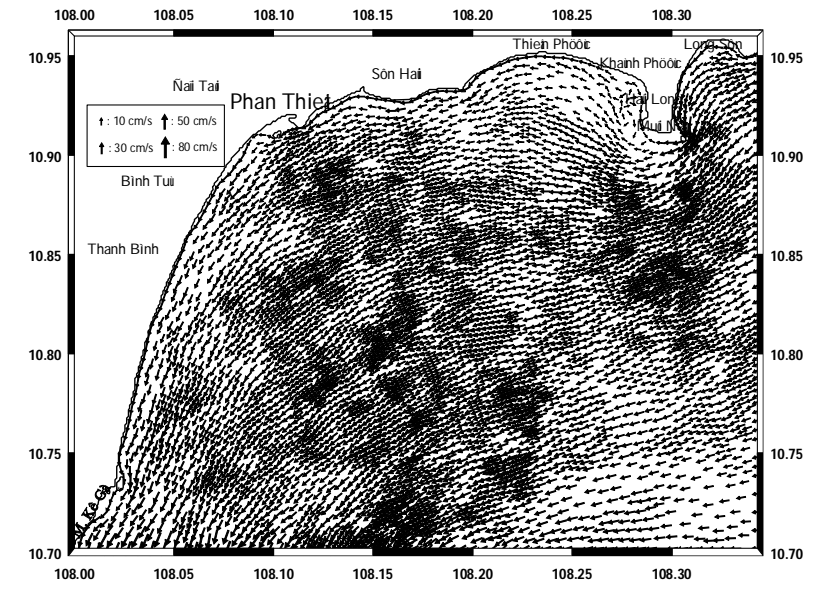

Hình 5b. Phân bố dòng chảy tổng hợp tại tầng mặt do ảnh hưởng trường gió mùa Đông Bắc 
Tại tầng 10m:

Tốc độ dòng ngang tại tầng $10 \mathrm{~m}$ đạt cực đại 40,4cm/s, hướng $193,1^{0}$; vị trí đạt cực đại $\left(108,0811^{0} \mathrm{E} ; 10,70595^{\circ} \mathrm{N}\right) ; \mathrm{H}=10,7 \mathrm{~m} ; \mathrm{A}_{\mathrm{H}}=3,18 \mathrm{~m}^{2} / \mathrm{s} ; \mathrm{T}=24,49^{0} \mathrm{C} ; \mathrm{S}=33,58^{0} \%$; vận tốc thẳng đứng $\mathrm{W}=-0,351 \mathrm{~cm} / \mathrm{s}$. Trong khi, dòng tổng hợp tại tầng $10 \mathrm{~m}$, có tốc độ dòng ngang tại tầng $10 \mathrm{~m}$ đạt cực đại $49,614 \mathrm{~cm} / \mathrm{s}$, hướng $197,107^{0}$; vị trí đạt cực đại $\left(108,0767^{0} \mathrm{E} ; 10,7014^{0} \mathrm{~N}\right) ; \mathrm{H}=25,2 \mathrm{~m}$; vận tốc thẳng đứng $\mathrm{W}=-0,513 \mathrm{~cm} / \mathrm{s}$.

Xét trên phương diện cực đại dòng thẳng đứng:

Tốc độ dòng hướng lên cao nhất có thể đạt $\mathrm{W}=+0,459 \mathrm{~cm} / \mathrm{s}$, tại vị trí $\left(108,1115^{\circ} \mathrm{E}\right.$; $\left.10,7044^{0} \mathrm{~N}\right)$, độ sâu $10,1 \mathrm{~m}$; độ nhớt rối nằm ngang $\mathrm{A}_{\mathrm{H}}=2,185 \mathrm{~m}^{2} / \mathrm{s}$; nhiệt độ nước biển $\mathrm{T}=$ $24,51^{\circ} \mathrm{C}$; độ mặn $\mathrm{S}=33,58 \%$; véc tơ vận tốc dòng theo phương ngang $(\mathrm{V}=29,8 \mathrm{~cm} / \mathrm{s}$, hướng $\left.190,6^{0}\right)$. Trong khi, tại dòng chảy tổng hợp, có tốc độ dòng hướng lên cao nhất có thể đạt $\mathrm{W}=+0,493 \mathrm{~cm} / \mathrm{s}$, tại vị trí $\left(108,1379^{\circ} \mathrm{E} ; 10,7015^{\circ} \mathrm{N}\right)$, độ sâu $16,3 \mathrm{~m}$; véc tơ vận tốc dòng theo phương ngang $\left(\mathrm{V}=38,4 \mathrm{~cm} / \mathrm{s}\right.$, hướng $\left.212,0^{\circ}\right)$

Tốc độ dòng hướng xuống cao nhất có thể đạt $\mathrm{W}=-0,985 \mathrm{~cm} / \mathrm{s}$, tại vị trí $\left(108,1418^{0} \mathrm{E} ; 10,7038^{0} \mathrm{~N}\right)$, độ sâu $25,1 \mathrm{~m}$; độ nhớt rối nằm ngang $\mathrm{A}_{\mathrm{H}}=0,285 \mathrm{~m}^{2} / \mathrm{s}$; nhiệt độ nước biển $\mathrm{T}=24,53^{\circ} \mathrm{C}$; độ mặn $\mathrm{S}=33,58 \%$; véc tơ vận tốc dòng theo phương ngang ( $\mathrm{V}$ $=37,1 \mathrm{~cm} / \mathrm{s}$; hướng $\left.=193,3^{0}\right)$. Trong khi, với dòng chảy tổng hợp thì tốc độ dòng hướng xuống cao nhất có thể đạt $\mathrm{W}=-1,04 \mathrm{~cm} / \mathrm{s}$, tại vị trí $\left(108,1485^{0} \mathrm{E} ; 10,7015^{0} \mathrm{~N}\right)$, độ sâu $32 \mathrm{~m}$; véc tơ vận tốc dòng theo phương ngang $\left(\mathrm{V}=25,3 \mathrm{~cm} / \mathrm{s}\right.$; hướng $\left.245,7^{0}\right)$. Chi tiết trên hình $6 \mathrm{a}, 6 \mathrm{~b}$.

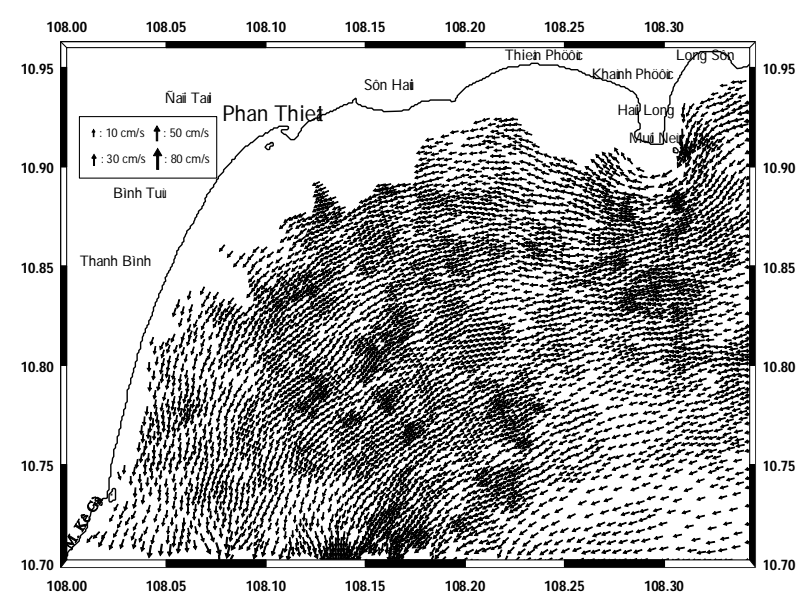

Hình 6a. Phân bố dòng chảy tại tầng $10 \mathrm{~m}$ vào trường gió mùa Đông Bắc

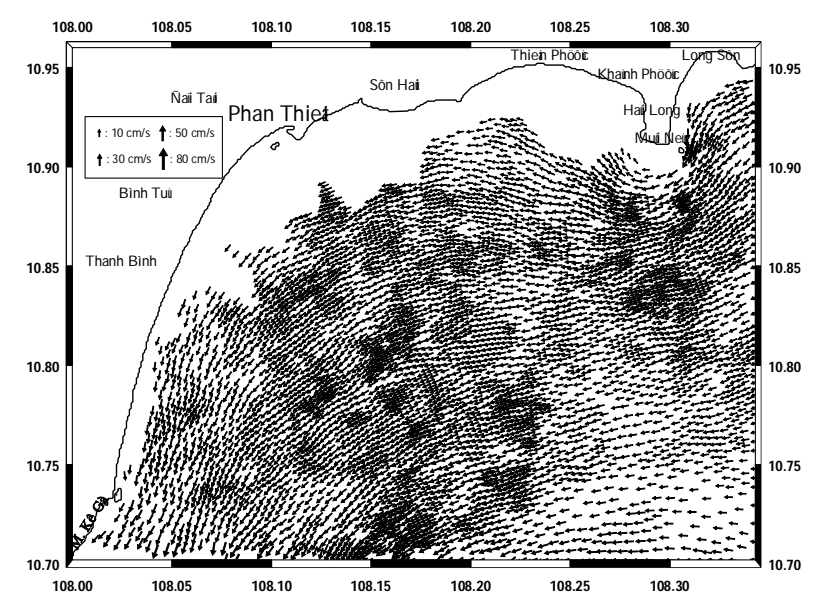

Hình 6b. Phân bố dòng chảy tổng hợp tại tầng $10 \mathrm{~m}$ do ảnh hưởng trường gió mùa Đông Bắc

\section{b. Đối với trường gió mùa Tây Nam}

\section{Tại tầng mặt:}

Theo tính toán thống kê trung bình, trong toàn bộ vùng nghiên cứu, tốc độ gió cực đại có thể đạt $7,9 \mathrm{~m} / \mathrm{s}$, hướng $64,1^{0}$ (tại vị trí $108,3428^{\circ} \mathrm{E} ; 10,9371^{\circ} \mathrm{N}$ ), trung bình $7,8 \mathrm{~m} / \mathrm{s}$ và cực tiểu $7,6 \mathrm{~m} / \mathrm{s}$, hướng $63,8^{0}$ (tại vị trí $108,0877^{\circ} \mathrm{E} ; 10,9060^{\circ} \mathrm{N}$ ). Theo tính toán phân bố 
dòng chảy tại tầng mặt, tốc độ dòng ngang đạt cực đạt $53 \mathrm{~cm} / \mathrm{s}$, hướng $10,3^{0}$; tại vị trí $\left(108,1086 \mathrm{E} ; 10,7015^{0} \mathrm{~N}\right)$; độ sâu $1,0 \mathrm{~m}$; độ nhớt rối nằm ngang $\mathrm{A}_{\mathrm{H}}=39,093 \mathrm{~m}^{2} / \mathrm{s}$, nhiệt độ $\mathrm{T}=28,50^{\circ} \mathrm{C}$, độ mặn $\mathrm{S}=33,19^{\circ} \%$. Trong khi, theo tính toán phân bố dòng chảy tổng hợp (có xét đến dòng dư) thì tại tầng mặt, tốc độ dòng ngang đạt cực đạt $36,6 \mathrm{~cm} / \mathrm{s}$, hướng $23,8^{0}$; tại vị trí $\left(108,0355^{\circ} \mathrm{E} ; 10,7054^{\circ} \mathrm{N}\right)$; độ sâu $9,1 \mathrm{~m}$. Chi tiết trên hình $7 \mathrm{a}, 7 \mathrm{~b}$.

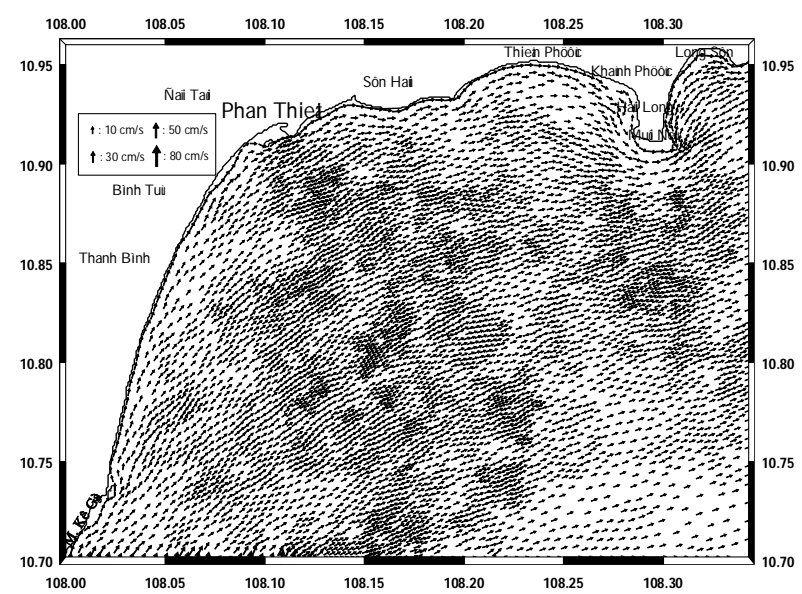

Hình 7a. Phân bố dòng chảy do gió tầng mặt vào trường gió mùa Tây Nam

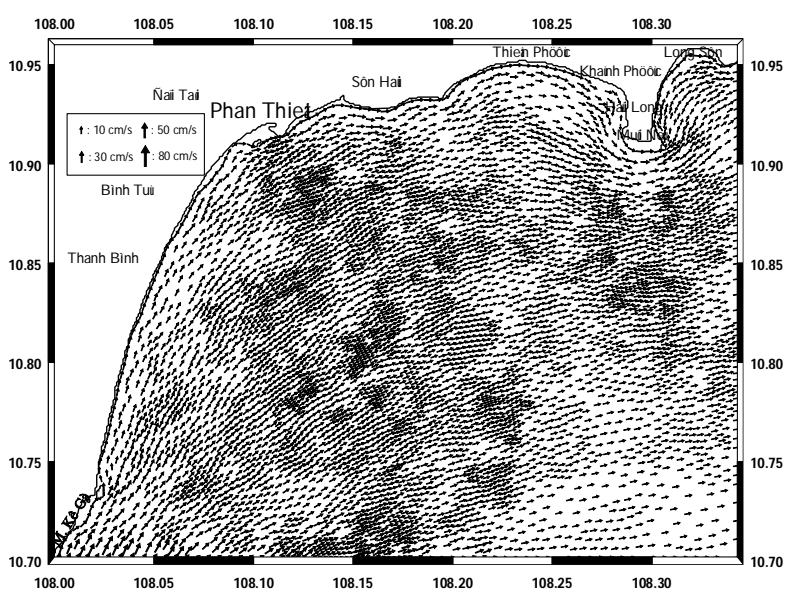

Hình 7b. Phân bố dòng chảy tổng hợp tại tầng mặt do ảnh hưởng trường gió mùa Tây Nam

Tại tầng 10m:

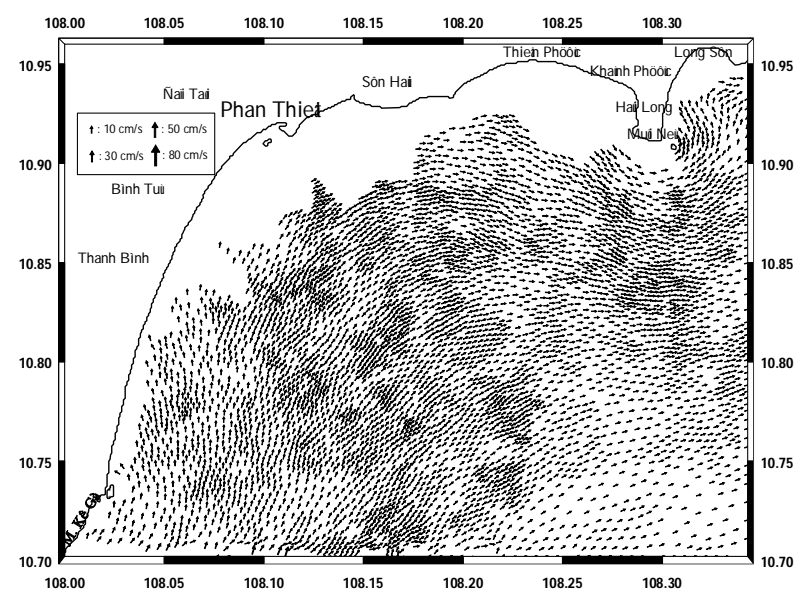

Hình 8a. Phân bố dòng chảy do gió tại tầng $10 \mathrm{~m}$ vào trường gió mùa Tây Nam

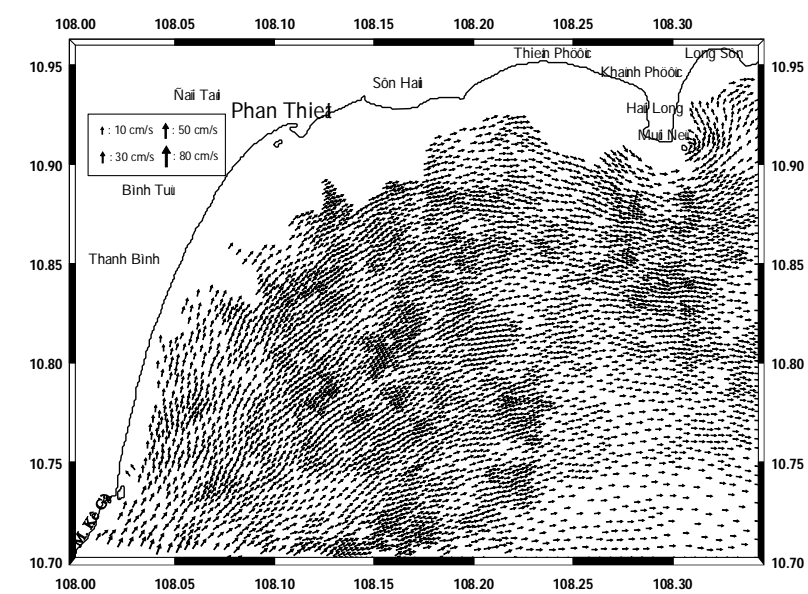

Hình $\mathbf{8 b}$. Phân bố dòng chảy tổng hợp tại tầng $10 \mathrm{~m}$ do ảnh hưởng trường gió mùa Tây Nam

Tốc độ dòng ngang tại tầng $10 \mathrm{~m}$ đạt cực đại $24,5 \mathrm{~cm} / \mathrm{s}$, hướng $16,9^{0}$; vị trí đạt cực đại $\left(108,1115^{0} \mathrm{E} ; 10,7044^{0} \mathrm{~N}\right) ; \mathrm{H}=10,1 \mathrm{~m} ; \mathrm{A}_{\mathrm{H}}=2,631 \mathrm{~m}^{2} / \mathrm{s} ; \mathrm{T}=28,23^{0} \mathrm{C} ; \mathrm{S}=33,28^{0} \%_{00} ;$ vận tốc thẳng đứng $\mathrm{W}=-0,351 \mathrm{~cm} / \mathrm{s}$. Trong khi, với dòng tổng hợp thì tốc độ dòng ngang đạt cực đại $28,5 \mathrm{~cm} / \mathrm{s}$, hướng $19,6^{0}$; vị trí đạt cực đại $\left(108,0418^{0} \mathrm{E} ; 10,7061^{0} \mathrm{~N}\right) ; \mathrm{H}=10,4 \mathrm{~m}$; vận tốc thẳng đứng $\mathrm{W}=-0,225 \mathrm{~cm} / \mathrm{s}$. 
Xét trên phương diện cực đại dòng thẳng đứng:

Tốc độ dòng hướng lên cao nhất có thể đạt $\mathrm{W}=+0,0802 \mathrm{~cm} / \mathrm{s}$, tại vị trí $\left(108,1698^{0} \mathrm{E}\right.$; $\left.10,7016^{0} \mathrm{~N}\right)$, độ sâu $36,4 \mathrm{~m}$; độ nhớt rối nằm ngang $\mathrm{A}_{\mathrm{H}}=0,103 \mathrm{~m}^{2} / \mathrm{s} ;$ nhiệt độ nước biển $\mathrm{T}=$ $28,20^{\circ} \mathrm{C}$; độ mặn $\mathrm{S}=33,27^{\circ} \%$; véc tơ vận tốc dòng theo phương ngang $(\mathrm{V}=2 \mathrm{~cm} / \mathrm{s}$, hướng $88,8^{\circ}$ ). Trong khi với dòng tổng hợp thì tốc độ dòng hướng lên cao nhất có thể đạt $\mathrm{W}=+$ $0,383 \mathrm{~cm} / \mathrm{s}$, tại vị trí $\left(108,1485^{0} \mathrm{E} ; 10,7015^{0} \mathrm{~N}\right)$, độ sâu $32 \mathrm{~m}$; véc tơ vận tốc dòng theo phương ngang $\left(\mathrm{V}=8,9 \mathrm{~cm} / \mathrm{s}\right.$, hướng $\left.77,5^{\circ}\right)$.

Tốc độ dòng hướng xuống cao nhất có thể đạt $\mathrm{W}=-0,351 \mathrm{~cm} / \mathrm{s}$, tại vị trí $\left(108,1115^{0} \mathrm{E} ; 10,7044^{0} \mathrm{~N}\right)$, độ sâu $10,1 \mathrm{~m}$; độ nhớt rối nằm ngang $\mathrm{A}_{\mathrm{H}}=2,631 \mathrm{~m}^{2} / \mathrm{s}$; nhiệt độ nước biển $\mathrm{T}=28,23^{\circ} \mathrm{C}$; độ mặn $\mathrm{S}=33,28 \%$; véc tơ vận tốc dòng theo phương ngang ( $\mathrm{V}$ $=24,5 \mathrm{~cm} / \mathrm{s}$; hướng $\left.16,9^{0}\right)$. Trong khi, theo tính toán dòng tổng hợp thì tốc độ dòng hướng xuống cao nhất có thể đạt $\mathrm{W}=-0,225 \mathrm{~cm} / \mathrm{s}$, tại vị trí $\left(108,0418^{0} \mathrm{E} ; 10,7062^{0} \mathrm{~N}\right)$, độ sâu $10,4 \mathrm{~m}$; véc tơ vận tốc dòng theo phương ngang $\left(\mathrm{V}=28,5 \mathrm{~cm} / \mathrm{s}\right.$; hướng $\left.19,6^{0}\right)$. Chi tiết trên hình $8 \mathrm{a}, 8 \mathrm{~b}$.

\section{THẢO LUẬN VÀ KẾT LUẬN}

Các kết quả nghiên cứu dòng dư (residual current) thấy có xuất hiện hai xoáy nghịch (cùng chiều kim đồng hồ) tại vị trí $\left(108,0565^{\circ} \mathrm{E}, 10,7214^{\circ} \mathrm{N}\right)$ gần mũi Kê Gà và một xoáy nhỏ hơn tại vị trí $\left(108,2806^{\circ} \mathrm{E}, 10,9011^{\circ} \mathrm{N}\right)$ gần Mũi Né. Vấn đề xuất hiện dòng xoáy này có thể do ảnh hưởng tính phi tuyến của sự thay đổi địa hình ven bờ, tuy nhiên vấn đề này cần phải nghiên cứu chi tiết hơn nữa để có câu trả lời hợp lý cho kết quả này.

Về các nghiên cứu cấu trúc dòng do ảnh hưởng của gió mùa, chúng tôi thấy rằng trong trường gió mùa Đông Bắc, tốc độ dòng ngang do gió lớn nhất có thể $85,3 \mathrm{~cm} / \mathrm{s}$, hướng $185,7^{0}$ xung quanh vị trí có tọa độ $\left(108,0554^{\circ} \mathrm{E} ; 10,7014^{\circ} \mathrm{N}\right)$; tuy nhiên kết quả phân tích dòng chảy tổng hợp (dòng triều, dòng chảy do gió, dòng mật độ) chỉ có thể đạt $60,6 \mathrm{~cm} / \mathrm{s}$, hướng $209,6^{0}$; tại vị trí $\left(108,0820^{\circ} \mathrm{E} ; 10,7015^{\circ} \mathrm{N}\right)$. Và đối với trường gió mùa Tây Nam, tốc độ dòng ngang do gió đạt cực đạt $53 \mathrm{~cm} / \mathrm{s}$, hướng $10,3^{0}$; tại vị trí $(108,1086 \mathrm{E}$; $10,7015^{0} \mathrm{~N}$ ) nhưng dòng chảy tổng hợp tốc độ dòng ngang đạt cực đạt $36,6 \mathrm{~cm} / \mathrm{s}$, hướng $23,8^{0}$; tại vị trí $\left(108,0355^{0} \mathrm{E} ; 10,7054^{0} \mathrm{~N}\right)$. Như vậy, trong vịnh Phan Thiết (vùng nghiên cứu) dòng tổng hợp thường nhỏ hơn dòng chảy do gió.

Từ các kết quả tính dòng chảy theo dòng dư, cho hai chế độ gió mùa và dòng tổng hợp bằng mô hình ba chiều phi tuyến dạng đầy đủ bằng phương pháp phần tử hữu hạn cho vùng nghiên cứu cho thấy khả năng ứng dụng phương pháp phần tử hữu hạn vào việc mô hình hóa chế độ dòng chảy tại các vùng nghiên cứu có địa hình phức tạp, biên mở rộng... Thông thường các kết quả tính theo phương pháp phần tử hữu hạn thường thu được tốc độ dòng chảy (xét trên toàn khu vực nghiên cứu) cao hơn so với sai phân hữu hạn, đó là do khả năng chi tiết hóa được chế độ dòng chảy tại khu vực biên ven bờ - đảo, nơi có cồn cát, bãi ngầm, ...[1, 2, 3, 4, 5]. Trong bài toán theo phương pháp phần tử hữu hạn, khi cần nâng cao độ chính xác nếu có các địa hình đường bờ chính xác, chúng tôi sẽ giải thêm từ $1 \mathrm{~m}$ (do bài toán ba chiều, chúng tôi chỉ giải bài toán có độ sâu tối thiểu là từ $1 \mathrm{~m}$ ) độ sâu trở vào bờ theo mô phỏng theo bài toán 2 chiều (trên cùng mạng lưới tính) với hai quá trình vật lý chính động lực học và động học (từ độ sâu $0,5 \mathrm{~m}$ vào đến độ sâu $0,1 \mathrm{~m}$ ), hy vọng có thể phản ánh khá tốt quy luật vật lý của quá trình lan truyền sóng dài trong những 
vùng nước rất nông. Trong khi ở phương pháp sai phân hữu hạn, bài toán đạt ổn định tốt tại các vị trí ven bờ thường phải có độ sâu lớn hơn $1 \mathrm{~m}$ [4]. Ngoài ra, vấn đề khoảng cách theo không gian của mạng lưới tính sai phân (ô lưới bằng nhau, hình vuông hoặc hình chữ nhật với kích thước tương đối lớn) nên đã có những mặt hạn chế khi đánh giá chế độ dòng chảy tại những biên bờ, điểm tính nhạy cảm (đảo chắn, bãi ngầm, ...), hoặc khi cần thể hiện chi tiết tính địa phương tại khu vực nghiên cứu. Từ những phân tích này, cho thấy triển vọng và hướng phát triển tương lai của phương pháp phần tử hữu hạn. Các dòng chảy tại dọc biên bờ và địa hình đáy phức tạp đã được thể hiện khá rõ trong nghiên cứu ở trên. Như vậy, với việc sử dụng phương pháp phần tử hữu hạn, chúng ta có thể dễ dàng tìm ra các vị trí nhạy cảm, vị trí có phân bố dòng chảy tương đối đặc biệt. Việc kiểm nghiệm tính đúng đắn của phương pháp phần tử với thực tế đo đạc sẽ giúp chúng ta hiệu chỉnh lại các thông số tính toán cần thiết, phục vụ tốt hơn cho mổ phỏng các bài toán dòng chảy trong biển. Nếu được chỉnh lý số liệu và đo đạc được thực hiện đồng bộ, chi tiết, đáng tin cậy với các dữ liệu đầu vào tốt, thiết nghĩ kết quả mô hình hóa bằng phương pháp phần tử hữu hạn sẽ mang tính định lượng cao. Các kết quả nghiên cứu đã cho thấy khả năng ứng dụng của mô hình, vấn đề còn lại kiểm tra và hiệu chỉnh mô hình cho các thực tế ứng dụng theo các hiện tượng mô phỏng khác nhau.

\section{TÀI LIỆU THAM KHẢO}

1. Bùi Hồng Long, Trần Văn Chung, 2007. Tính toán dòng triều tại cụm đảo Song Tử bằng phương pháp phần tử hữu hạn. Tuyển tập báo cáo hội nghị Quốc gia "Biển Đông2007”, 735-750.

2. Bùi Hồng Long, Trần Văn Chung, 2008. Kết quả mô phỏng chế độ vịnh Cam Ranh bằng phương pháp phần tử hữu hạn. Tạp chí Khoa học và Công nghệ biển, 4(8), 19-35.

3. Bùi Hồng Long, Trần Văn Chung, 2008. Modelling material transport in North Danger Reff, the Spratlys, based on three-dimensional nonlinear finite element model for wind currrents. Proceedings on the Conference on the results of the PhilippinesVietnam joint oceanographic and marine scientific research expedition in the South China Sea (JOMSRE-SCS I to IV), 135-147.

4. Bùi Hồng Long, Trần Văn Chung, 2009. Tính toán dòng chảy trong khu vực nước trồi Nam Trung bộ bằng mô hình dòng chảy ba chiều (3-D) phi tuyến. Tạp chí Khoa học và Công nghệ biển, 2(9), 1-25.

5. Bùi Hồng Long, Trần Văn Chung, 2009. Nghiên cứu chế độ dòng dư tại vùng biển Nam - Trung bộ Việt Nam. Tạp chí Khoa học và Công nghệ biển, 4(9), 5-24.

6. Blumberg, A.F., Galperin, B., O'Connor, D.J., 1992. Modelling vertical structure of open-channel flows. ASCE, In Journal of Hydraulic Engineering, 118, 1119-1134.

7. Elliott, A.J., Wilkins, B.T., Mansfield, P., 2001. On the disposal of contaminated milk in coastal waters. Marine Pollution Bulletin, 42, 927-934.

8. Gómez-Gesteira, M., Montero, P., Prego, R., Taboada, J.J., Leitao, P., RuizVillareal, M., Neves, R., Perez-Villar, V., 1999. A two-dimensional particle-tracking model for pollution dispersion in A Coru ns and Vigo Rias (NW Spain). Oceanologica Acta, 22, 167-177. 
9. Large, W., Pond, S., 1981. Open Ocean momentum flux measurements in moderate to strong winds. J. Phys. Oceanogr., 11, 324-336.

10. Lynch, D.R., Naimie, C.E., 1993. The $\mathrm{M}_{2}$ Tide and Its Residual on the Outer Banks of the Gulf of Maine, J.Phys Oceanogr 23, 2222-2253.

11. Mellor, G.L., Yamada, T., 1982. Development of a turbulent closure model for geophysical fluid prolems. Reviews of Geophysics and Space Physics, 20(4), 851-875.

12. Nakano, M., Povinec, P., 2003. Oceanic general circulaton model for the assessment of the distribution of ${ }^{137} \mathrm{Cs}$ in the world ocean. Deep-Sea Research II, 50, 2803-2816.

13. Periáriez, R. , Elliott, A.J., 2002. A particle tracking method for simulating the dispersion of non-conservative radionuclides in coastal waters. Journal of Environmental Radioactivity, 58, 13-33.

14. Periáriez, R., Pascual-Granged, A., 2008. Modelling surface radioactive, chemical and oil spills in the Strait of Gibraltar. Computers \& Geosciences, 34, 163-180.

15. Pingree, R.D., Maddock, L., 1977. Tidal residuals in the English Channel. J mar biol Ass UK 57, 339-354.

16. Polovina, J.J., Kleiber, P., Kobayashi, D.R., 1999. Application of Topex-Poseidon satellite altimetry to simulate transport dynamics of larvae of spiny lobster, Panulirus marginatus in the Northwestern Hawaiian Islands, 1993-1996. Fish. Bull, 97, 132-143.

17. Prandle, D., 1997. Tidal and Wind-driven Currents From OSCR . Oceanography, 10(2), 57-59.

18. Prandle, D., Layer, R., 1993. Residual Curent Through the Dover Strait Measured by H.F Radar . Estuary, Coastal and Shelf Sciences (Print) 37, 635-653, Elvier.

19. Schonfield, W., 1995. Numerical simulation of the dispersion of artificial radionuclides in the English Channel and the North Sea. Journal of Marine Sysems, 6, 529-544.

20. Stentchev, A., Korotenko, K., 2005. Dispersion processes and transport pattern in the ROFI system of the eastern English Channel derived from a particle-tracking model. Journal of Marine Systems, 25, 2294-2304.

21. Trenberth, K.E., Large, W.G., Olson, J.G., 1990. The mean annual cycle in global ocean wind stress. J. Phys. Oceanogr., 20, 1742-1760.

22. Valle-Levinson A., Moraga J., Olivares J., Blanco J.L., 2000. Tidal and Residual Circulation in Semi-avid Bay: Coquimbo Bay, Chile. Continental Shelf Research, 20(15), 2009-2028.

23. Walters, R.A., 1986. A finite element model for tidal and residual circulation. Communications in applied numerical mothods, 2, 393-398. 


\section{STUDY ON THE CURRENT REGIME IN PHAN THIET BAY USING 3D NONLINEAR FINITE ELEMENT METHOD}

\section{BUI HONG LONG, TRAN VAN CHUNG}

Summary: Different types of currents (residual-, tidal-, wind-and general currents, etc.) in Phan Thiet bay have been studied using $3 D$ nonlinear finite element method (FEM). The studied results showed the applicability of FEM for simulation of current regime in waters with complex topography and large liquid boundary.

To evaluate the applicability of the model, we calculated separately the 5 main current constituents $M_{2}, S_{2}, K_{1}, O_{1}, N_{2}$ of tidal currents. Then the harmonic constants of these current constituents were compared with data collected from Phan Thiet water level station. According to the comparison results, the absolute differences are as follows: the amplitude was $\max 3.1 \mathrm{~cm}$ (constituent $M_{2}$ ), $\min 0.0 \mathrm{~cm}$ (exact constituent $S_{2}$ ), the tidal phase was max $13.9^{\circ}$ (constituent $K_{1}$ ) and min $3.8^{0}$ (constituent $S_{2}$ ).

In the surface layer, during northeast monsoon period, the horizontal wind currents may reach its max speed of $85.3 \mathrm{~cm} / \mathrm{s}$, direction $185.7^{\circ}$ around the position $\left(108.0554^{\circ} \mathrm{E}\right.$; $\left.10.7014^{0} \mathrm{~N}\right)$; but the peak horizontal speed of the general currents can reach $60.6 \mathrm{~cm} / \mathrm{s}$, direction $209.6^{\circ}$, around position $\left(108.0820^{\circ} \mathrm{E} ; 10.7015^{\circ} \mathrm{N}\right)$. During southwest monsoon period, max speed of the horizontal wind currents reaches $53.0 \mathrm{~cm} / \mathrm{s}$, direction $10.3^{0}$; position $\left(108.1086 \mathrm{E} ; 10.7015^{\circ} \mathrm{N}\right)$, whereas, general current was $36.6 \mathrm{~cm} / \mathrm{s}$, direction $23.8^{0}$; position $\left(108.0355^{\circ} \mathrm{E} ; 10.7054^{\circ} \mathrm{N}\right)$.

These study results are the first $3 D$ nonlinear calculation results for the currents in Phan Thiet bay. These results have shown the possibility of detailed study of current distribution and finding out positions with particular current distribution in this area, providing scientific base and reliable information for a reasonable development planning.

Ngày nhận bài: 14 - 1 - 2012

Người nhận xét: TS. Lê Đình Mầu 